\title{
DIE ÖSTERREICH-BIBLIOTHEK IN ZAGREB (AUSTRIJSKA KNJIŽNICA ZAGREB) ${ }^{1}$
}

\section{von Blaženka Klemar Bubić}

Zusammenfassung: Die Österreich-Bibliothek am Standort der Philosophischen Fakultät in Zagreb wurde im Juni 2015 auf Initiative der Österreichischen Botschaft, des Österreichischen Kulturforums Zagreb und der Abteilung für Germanistik eröffnet. Der Bibliotheksbestand ist nicht im Besitz der Bibliothek der Philosophischen Fakultät, sondern hat den Status einer Dauerleihgabe und wird gemäß den Vorschriften der Bibliothek der Philosophischen Fakultät den Benutzer*innen zur Verfügung gestellt. Die erste Österreich-Bibliothek wurde 1986 in Krakau eröffnet. Dank der Initiative des damaligen österreichischen Außenministers Dr. Alois Mock wurden mehrere Österreich-Bibliotheken auf dem Gebiet der ehemaligen Habsburgermonarchie gegründet. Diese erleichtern ihren Benutzer*innen den Zugang zu österreichischer Literatur, Wissenschaft, sowie zu Informationen aus Österreich. Darüber hinaus leisten sie einen großen Beitrag zur Verbreitung der deutschen Sprache und österreichischen Kultur und dienen der Förderung und Vertiefung der kulturellen und wissenschaftlichen Zusammenarbeit. Die neue Sammlung stellte für die Bibliothek der Philosophischen Fakultät eine große Bereicherung dar und obwohl sie einerseits zahlreiche Verpflichtungen mit sich brachte und bringt, eröffnet sie andererseits auch viele Möglichkeiten zur Zusammenarbeit mit kulturellen und wissenschaftlichen Institutionen in Österreich.

Schlüsselwörter: Bibliothek der Philosophischen Fakultät Zagreb; Österreich-Bibliothek Zagreb; Bibliotheken zwischen Unterhaltsträger*innen und Benutzer*innen; Spenden

\section{THE AUSTRIAN LIBRARY IN ZAGREB (AUSTRIJSKA KNJIŽNICA ZAGREB)}

Abstract: The Austrian Library at the the faculty of the Humanities and Social Sciences of the University of Zagreb was opened in June 2015 on the initiative of the Austrian Embassy, the Austrian Cultural Forum Zagreb and the Department of German Studies. The library is not owned by the library of the Humanities and Social Sciences but has the status of a permanent loan and is made available to users according to the regulations of the library of the faculty. The first Austrian Library was opened in 1986 
in Krakow. Thanks to the initiative of the then Austrian minister for foreign affairs Alois Mock were founded several Austrian Libraries in the area of the former Habsburg monarchy. These facilitate their users' access to Austrian literature, scholarship, and information from Austria. Also, they make a major contribution to the spread of the German language and Austrian culture and serve to promote and deepen cultural and scientific cooperation. The new collection was a great enrichment to the library of the faculty, and although it has many obligations, it also opens many opportunities for cooperation with cultural and scientific institutions in Austria.

Keywords: Library of the faculty of the Humanities and Social Sciences of the University of Zagreb; Austrian Library Zagreb; libraries between funding bodies and users; donations

DOI: https://doi.org/10.31263/voebm.v72i1.2193

(C) Blaženka Klemar Bubić

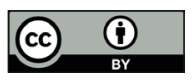

Dieses Werk ist lizenziert unter einer

Creative-Commons-Lizenz Namensnennung 4.0 International

\section{Inhalt}

\section{Einleitung}

2. Österreich-Bibliotheken im Ausland

3. Österreich-Bibliotheken in Kroatien

4. Die Geschichte der Österreich-Bibliothek Zagreb

5. Schlussbemerkungen 


\section{Einleitung}

Diese Arbeit strebt danach die Österreich-Bibliothek darzustellen: ihre Vergangenheit, Entstehungsgeschichte und Entwicklung sowohl wie auch ihre Einordnung in einen breiteren gesellschaftlichen Kontext. Auf die guten Beziehungen zwischen Österreich und Kroatien weisen zahlreiche Fakten hin, wie zum Beispiel: der Name der Bibliothek, die geografische Lage bzw. die Tatsache, dass österreichische Kulturinstitute und österreichische Bibliotheken im Ausland meistens in den Gegenden gegründet wurden, wo die größte Anzahl der österreichischen Minderheit angesiedelt ist. Die gemeinsame Geschichte der Republik Kroatien bzw. der Stadt Zagreb mit Österreich, die Jahrhundertelang Teil der Habsburgermonarchie, des Kaiserreichs Österreich bzw. Österreich-Ungarns war, ist heute noch deutlich im kulturellen Erbe und der regionalen Identität der Stadt Zagreb sichtbar, so etwa auf sprachlicher Ebene - wie im Vorwort von „Agramer“, dem Wörterbuch der deutschen Lehnwörter in der Stadtsprache von Zagreb, zu lesen ist: „Zagreb war schon immer ein Ort, wo Sprache und Kultur aufeinandertreffen.“2 „Besonders stark war die Beziehung zwischen Zagreb und Wien, nicht nur im politischen Sinne, im mitteleuropäischen Umfeld handelte es sich auch um kulturelle Anstöße "3; diese Verbindungen dauern an, wie sich auch an der Anzahl der deutschsprachigen Bevölkerung in Zagreb nachvollziehen lässt (Volkszählung aus dem Jahr 2011: 104 Österreicher*innen und 364 Deutsche $^{4}$ ). In einer Großstadt wie Zagreb kann man trotz der historischen Verbindungen nicht bzw. nicht mehr davon ausgehen, dass es sich um eine Bibliothek speziell für die österreichische und deutsche Minderheit handelt, wie das beispielsweise bei der Österreich-Bibliothek in Osijek der Fall ist, die als Zentralbibliothek der Österreicher*innen institutionell an die Stadt und Universitätsbibliothek Osijek angegliedert ist und ihr Selbstverständnis aus der Tradition der Literaturversorgung einer autochtonen deutschsprachigen Bevölkerungsgruppe gewinnt. ${ }^{5}$

Bei einer ausführlicheren Analyse der Benutzer*innen der Bibliothek und Lesehalle des Österreichischen Kulturinstituts, das später in Österreichisches Kulturforum umbenannt wurde, offenbart sich die Veränderung deutlich. Darüber hat sich auch die Bibliothekarin des Österreichischen Kulturforums geäußert: „Die Angehörigen der österreichischen und deutschen Minderheit waren am zahlreichsten während der 60er, 70er und 80er Jahre, indessen ist ihre Anzahl seit den 90ern bis zum Jahr 2005 sichtbar gesunken. Während der letzten zehn Jahre (von 2005 bis 2015) der Tätigkeit der Bibliothek und Lesehalle, die zu dieser Zeit in der Gundulićeva-Straße angesiedelt war, hat nur eine geringe Zahl der Angehörigen der beiden Minderhei- 
ten diese besucht. Der Grund dafür ist hauptsächlich das fortgeschrittene Alter der Angehörigen der österreichischen oder deutschen Minderheit, die die deutsche Sprache aktiv gebrauchen. Sie sind entweder gestorben oder alt geworden."

Die ersten Initiativen zur Gründung der Österreich-Bibliothek Zagreb wurden schon 2007 vom damaligen Leiter des Österreichischen Kulturforums Christian Ebner durch die Einladung des Vertreters der Abteilung für Germanistik der Philosophischen Fakultät in Zagreb und der Leiterin der Bibliothek der Abteilung für Germanistik zu einem Treffen der deutschen Bibliotheken in Kroatien (30. November-1. Dezember 2007) in Gang gesetzt, doch die von ihm angestoßenen Bemühungen wurden erst im Juni 2015 mit Erfolg gekrönt.

Die Österreich-Bibliothek Zagreb ist eine Hochschul- bzw. Fakultätsbibliothek, die sich mit ihren Inhalten, Leistungen und Programmen vollständig ihren Studierenden und Wissenschaftler*innen gewidmet hat. „Hochschulbibliotheken sind Institutionen, die Dokumente und Informationen mit Wissenschafts-, Bildungs- oder allgemeinem Kulturcharakter sammeln, bearbeiten, verwahren und zur Benutzung freigeben. Hochschulbibliotheken sind ein wesentlicher Bestandteil der Infrastruktur der Wissenschaft, Bildung und Forschung ${ }^{7 \text { “ }}$ und ihr Aufbau erfordert, „dass man den Bedürfnissen und Anforderungen ihrer Benutzer*innen folgt" und "die wissenschaftlichen und auf die Lehre bezogenen Erfordernisse der zugehörigen Abteilung weiterentwickelt"

Die strategische Entscheidung, bei der Ausrichtung der Bibliothek im Zuge ihrer Neugründung den Fokus nicht auf die schwindenden deutschsprachigen Minderheiten zu legen, sondern die Einrichtung einer Sammlung mit akademischem Zielpublikum zu verfolgen, erwies sich im Nachhinein als: Es wurde seit Gründung nicht ein ${ }^{*}$ einzige ${ }^{*} r$ Benutzer* in aus der demographisch mittlerweile vernachlässigbaren ersteren Gruppe verzeichnet.

\section{2. Österreich-Bibliotheken im Ausland}

Die erste Österreich-Bibliothek wurde 1986 in der polnischen Stadt Krakau eröffnet. Der Außenminister der Republik Österreich zu jener Zeit, Dr. Alois Mock, begann nach den politischen Veränderungen in Europa im Jahre 1989 damit, systematisch ein Programm für Deutschlektor*innen vorzubereiten, mit dem Ziel die Sprache zu verbreiten und die kulturellen Beziehungen mit Österreich zu stärken. Auch um diesem Programm 
Unterstützung zu leisten, eröffnete er in Folge dessen weitere ÖsterreichBibliotheken in Universitätsstädten ohne Österreichische Kulturforen. ${ }^{10}$

In den letzten dreißig Jahren, d.h. seit 1986, wurden 65 Österreich-Bibliotheken in 28 Städten rund um die Welt gegründet. Nach dem Bericht ${ }^{11}$ für das Jahr 2015 beträgt der gesamte Bestand der Österreich-Bibliotheken 432.000 Exemplare. Die Österreich-Bibliotheken haben kumuliert ungefähr 388.000 Besucher*innen pro Jahr und organisieren etwa tausend unterschiedliche kulturelle und wissenschaftliche Veranstaltungen wie Konferenzen, Workshops, Büchervorstellungen, Wettbewerbe, Ausstellungen, Filmabende usw.

Die meisten Österreich-Bibliotheken befinden sich auf dem Gebiet der ehemaligen Habsburgermonarchie, jedoch haben sie sich durch die Zeit auch außerhalb dieses Gebiets verbreitet - auch außerhalb von Europa nach Mittelasien und den Nahen Osten. Die geographische Verortung der Bibliotheken liegt in einem Gebiet zwischen Sankt Petersburg (Russische Föderation) im Norden und Jerusalem (Israel) im Süden sowie Trento/Trient (Italien) im Westen und Bischkek (Kirgistan) im Osten (Abbildung 1).

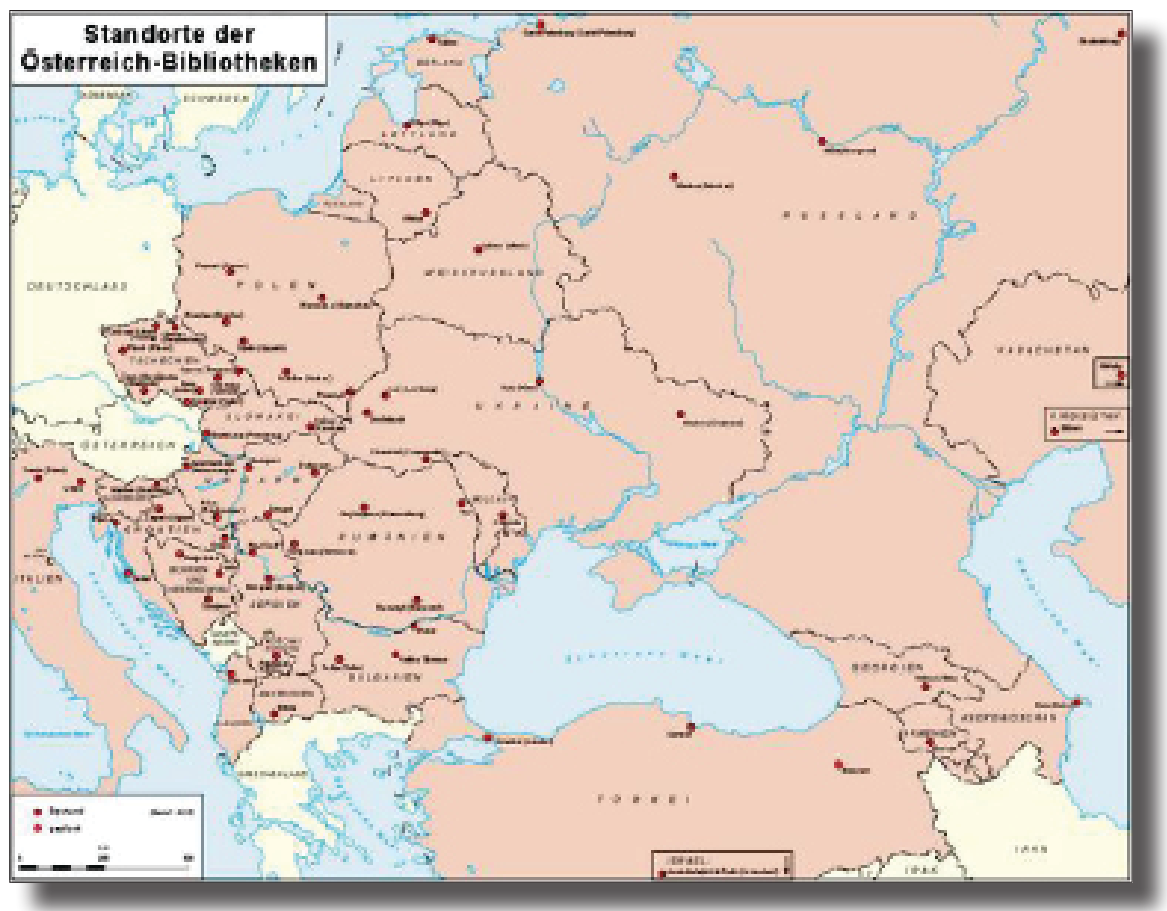

Abb. 1: Karte der Österreich-Bibliotheken im Ausland (Bild: BMEIA) ${ }^{12}$ 
Die höchste Anzahl an Österreich-Bibliotheken befindet sich in der Tschechischen Republik, mit insgesamt neun, in Polen gibt es sechs, in Ungarn und der Ukraine fünf, in der Russischen Föderation und in Kroatien vier, in Bosnien-Herzegowina, Bulgarien und Rumänien drei, in der Slowakei, Italien, Serbien und der Türkei zwei, und in den restlichen Ländern (Albanien, Armenien, Aserbaidschan, Belarus, Estland, Georgien, Israel, Kasachstan, Kirgistan, Kosovo, Lettland, Litauen, Mazedonien, Republik Moldau und Slowenien) jeweils eine Österreich-Bibliothek (Abbildung 2).

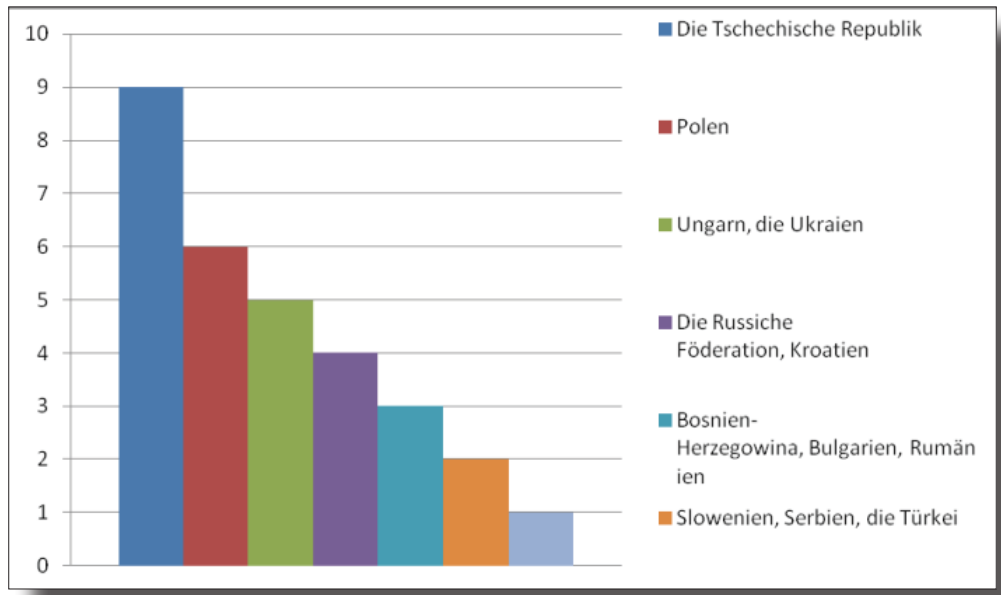

Abb. 2: Anzahl der Österreich-Bibliotheken nach Ländern ${ }^{13}$

Das Hauptziel der Österreich-Bibliotheken besteht in der Verbreitung der österreichischen Literatur, Wissenschaft und Kultur - was neben dem Fokus auf den Buchbestand auch eine Vertiefung etwa in Form von Veranstaltungen erlaubt und auch einen flexiblen und innovativen kulturpolitischen Einsatz ermöglicht -, sowie in der Erleichterung des Zugangs zu Informationen aus Österreich. Die Bibliotheken sind öffentlich zugänglich und es gibt keine Beitrittsgebühren. Den Großteil der Benutzer*innen machen das wissenschaftliche und Lehrpersonal, Studierende und Angehörige der deutschsprachigen Minderheiten aus. Die Sektion V (Kultur) des österreichischen Bundesministeriums für Europa, Integration und Äußeres (BMEIA) ist mit Unterstützung der lokalen Botschaften und Österreichischen Kulturforen verantwortlich für die Organisation und Koordination der Österreich-Bibliotheken im Ausland. Sie finanziert die Erwerbung neuer Bestände, während sich die Gasteinrichtungen, in denen sich die Bibliotheken befinden, um die Infrastruktur und das Personal kümmern. Für jede Bibliothek ist eine wissenschaftliche Betreueriln zuständig, 
meistens eine Hochschulprofessoriln und Germanistiln, die sich mit dem Themenbereich der österreichischen Kultur oder Sprache befasst, eine Bibliothekarin und eine österreichische Lektorin, die über das Lektor*innenprogramm des Österreichischen Austauschdiensts (ÖAD) die Bibliotheken mitbetreuen.

Auf der Webseite Österreich-Bibliotheken im Ausland (http://www.oesterreich-bibliotheken.at/) befindet sich eine Plattform, die zur Vernetzung der Österreich-Bibliotheken im Ausland sowie zur Verwaltung der Bestellungen, Rechnungen, Berichte, Ankündigungen und Archive der Veranstaltungen dient. Dort finden sich alle Informationen über die Geschichte und Projektziele der Österreich-Bibliotheken im Ausland. Auf dieser Webseite befindet sich auch eine Liste der Österreich-Bibliotheken, die die Besucher*innen zu den jeweiligen Webseiten weiterleitet, wo sie Informationen über die jeweilige Bibliothek, ihre Entstehungsgeschichte, eine Beschreibung des Bestandes, Kontaktinformationen, den Katalog der Bibliothek, Webseiten und Facebook-Seiten finden können. An der Erstellung eines übergreifenden Katalogs als „virtuelle Bibliothek“ wird noch gearbeitet.

Die Standorte, an denen sich die Österreich-Bibliotheken befinden, sind überwiegend National- oder Universitätsbibliotheken, seltener, wie es bei der Österreich-Bibliothek Zagreb der Fall ist, Fakultätsbibliotheken. Doch auch öffentliche Bibliotheken, Bibliotheken von Vereinen, Verbänden und Zentren sowie Bibliotheken der Akademien der Wissenschaften dienen als Gastinstitutionen. Eine Aufteilung nach Gastinstitutionen zeigt die Abbildung 3.

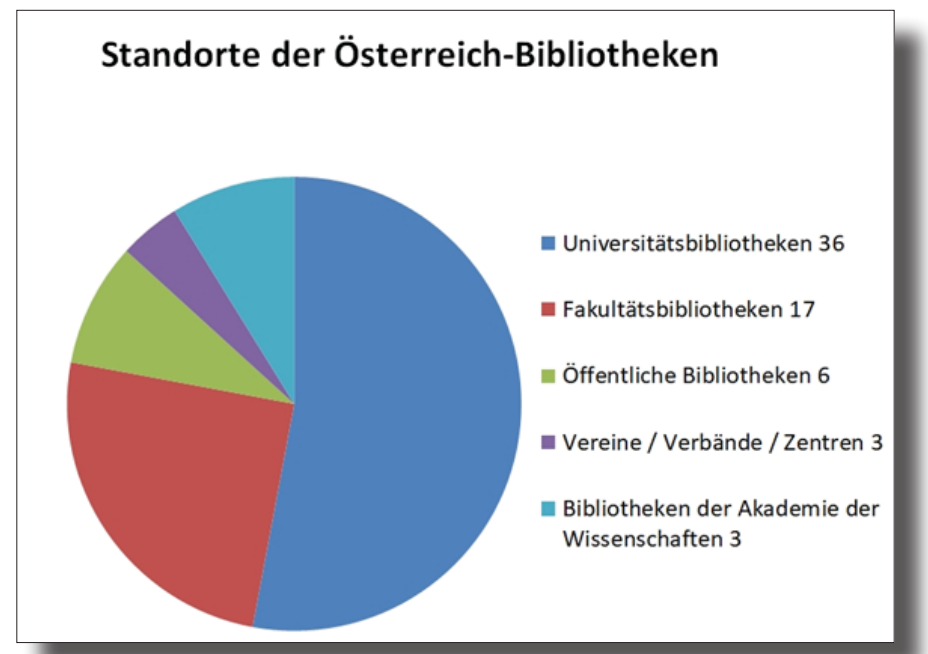

Abb. 3: Standorte der Österreich-Bibliotheken ${ }^{14}$ 


\section{3. Österreich-Bibliotheken in Kroatien}

Die Eröffnung einer Österreich-Bibliothek ist ein Zeichen der engen Beziehung zwischen Österreich und Kroatien. Österreich-Bibliotheken werden mit einem Bestand von 3.500 repräsentativer Werke und Periodika ausgestattet, wobei die Weiterentwicklung von der spezifischen Umgebung abhängt, in der sich die Bibliothek befindet. ${ }^{15}$ Alle Bibliotheksstücke, der Basisbestand sowie auch der weitere Erwerb, sind ein Geschenk des Ministeriums der Republik Österreich. Die Werke der Österreich-Bibliothek gehen nicht in den Bestand der Gastinstitution über, sondern es wird dafür ein eigenes Inventarbuch geführt.

In Kroatien gibt es vier Österreich-Bibliotheken. Die erste Österreich-Bibliothek bzw. die Österreichische Lesehalle in Kroatien wurde am 19. Oktober 1995 in Osijek eröffnet (Österreich-Bibliothek Osijek), und zwar als eine eigene Abteilung der Stadt- und Universitätsbibliothek Osijek. Nach den Daten der Volkszählung aus dem Jahr 2001 lebte ein Drittel aller Deutschen in Kroatien in der Gespanschaft Osijek-Baranja. ${ }^{16}$ Die Autorin Marija Erl Šafar hat diesen Umstand auch begründet: „wegen der historischen und kulturellen Beziehung von Slawonien und Österreich von 1687 bis $1918^{\text {"17 }}$ sei es selbstverständlich, dass die erste Österreich-Bibliothek, die auch die Zentralbibliothek der Deutschen und Österreicher*innen in der Republik Kroatien ${ }^{18}$ ist, gerade in Osijek (von Vlado Obad als „die älteste deutsche Stadt im Südosten" ${ }^{19}$ bezeichnet) eröffnet wurde. Auf dem Gebiet der Gespanschaft Osijek-Baranja wirken sechs Gesellschaften, die für das kulturelle Leben der österreichischen und deutschen Minderheit sorgen, eine von ihnen ist die Kroatisch-österreichische Gesellschaft, die in der Österreich-Bibliothek Osijek ihren Sitz hat. ${ }^{20}$ Der Bestand der Österreichischen Lesehalle umfasst ungefähr 8.000 Titel und entspricht den Anforderungen des Standortes mit der Verbindung der Tätigkeit einer öffentlichen Bibliothek und den Herausforderungen der Universitätstätigkeit. Wie auch in anderen öffentlichen Bibliotheken sind verschiedene Genres für alle Altersgruppen vertreten, jedoch mit Fokus auf österreichischer Literatur und Geschichte sowie Nachschlagewerke (Wörterbücher, Lexika, Grammatiken und Enzyklopädien), was ihre Rolle als Universitätsbibliothek, aber auch multikulturelle Bibliothek unterstreicht. ${ }^{21}$ In der Bibliothek oder in Zusammenarbeit mit dieser werden - mit Unterstützung des Österreichischen Kulturforums und lokalen Institutionen aus dem Kulturleben, wie zum Beispiel dem Kroatischen Nationaltheater, dem Archäologischen Museum, der Galerie der bildenden Künste, dem Kinoverband Osijek, der Abteilung für Germanistik der Philosophischen Fakultät der Universität in Osijek, vielen Grund- und Mittel- 
schulen und Kindergärten, in denen Deutsch gelernt wird - Literatur- und Musikabende, Vorlesungen, Ausstellungen, Filmausstrahlungen, Buchpräsentationen, Schüler*innen- und Studierendenbesuche und Workshops, anlässlich bestimmter Gedenktage u.a. organisiert. Die Besucher*innen sind meist die Angehörigen der österreichischen und deutschen Minderheiten, Studierende und Lehrkräfte der Philosophischen Fakultät in Osijek, vorzugsweise der Germanistik sowie Pensionist*innen, Schüler*innen und Vorschüler*innen, die Deutsch lernen. ${ }^{22}$

Die zweite Österreich-Bibliothek in Kroatien wurde am 23. September 2011 an der Philosophischen Fakultät in Rijeka eröffnet (Österreich-Bibliothek Rijeka). Diese ist innerhalb der Philosophischen Fakultät, genauer an der Germanistikabteilung beheimatet. Der Bibliotheksbestand beläuft sich ungefähr auf 4000 Titel aus den Bereichen der Literatur, Linguistik, Geschichte, Psychologie, Soziologie und schließt auch eine kleinere Sammlung rezenter Periodika ein. Die Hochschul- bzw. Fakultätsbibliothek erleichtert den Zugang zu Literatur und Informationen aus Österreich und organisiert zusammen mit dem Österreichischen Kulturforum Zagreb zahlreiche kulturelle Veranstaltungen wie Literatur- und Filmabende oder Ausstellungen. Diese Ereignisse sind oft zweisprachig gestaltet, sodass mehr Besucher* innen angezogen werden. In Zusammenarbeit mit den österreichischen Universitäten werden auch fachliche und wissenschaftliche Workshops sowie Vorlesungen abgehalten, die an das wissenschaftliche Personal, Lehrpersonal und Studierende der Germanistik gerichtet sind. Mit dem Ziel, die deutsche Sprache zu fördern, wurden auch Projekte gestartet, im Zuge derer auch Germanistikstudent* innen volontieren. ${ }^{23}$

Als Drittes wurde die Österreich-Bibliothek „Dr. Alois Mock“ am 1. Juli 2013 an der Universitätsbibliothek in Zadar eröffnet. Die Bibliothek besitzt einen Bestand von ungefähr 2500 Titeln und eine geringe Zahl von rezenten Periodika wie zum Beispiel Manuskripte, Österreich-Spiegel usw., sowie auch digitale Medien. Der Schwerpunkt des Bibliotheksbestandes liegt auf dem Gebiet der österreichischen Literatur, Literaturtheorie und Linguistik, daneben sind Bücher zu den Themen Philosophie, Geschichte, österreichische Politik, Architektur, Kunst, Methodik des Deutschunterrichts, Kunstgeschichte, Musik und auch ein paar Kinderbücher zu finden. Es handelt sich um eine Hochschul- bzw. Fakultätsbibliothek, deren Bestand allen frei zugänglich ist; eine Entlehnung ist allerdings nur den Angehörigen der Universität Zadar möglich. Zusammen mit der Germanistikabteilung und dem Österreichischen Kulturforum Zagreb organisiert die Bibliothek für Studierende, aber auch Schüler*innen, die Deutsch lernen, unter anderem Literatur- und Filmabende, Workshops, Vorlesungen, Ausstellungen und Musikabende. ${ }^{24}$ 


\section{Die Geschichte der Österreich-Bibliothek Zagreb}

Vor mehr als 60 Jahren wurde in Zagreb die Österreichische Lesehalle gegründet. Nach dem Zweiten Weltkrieg und dem österreichischen Staatsvertrag begann das Bundesministerium für Bildung damit, Kulturinstitute in westlichen Ländern und auf dem Gebiet der ehemaligen Habsburgermonarchie zu gründen. Im Zuge dessen wurde im Jahr 1955 auch die Österreichische Lesehalle in der Gundulićeva-Straße eröffnet. In der Sozialistischen Föderativen Republik Jugoslawien war Deutsch keine besonders beliebte Sprache. Hinsichtlich der jahrhundertelangen historischen, kulturellen und wirtschaftlichen Beziehungen zwischen Kroatien und Österreich und betreffend dem Interesse an allen Segmenten der österreichischen Kultur, von denen Kroatien nach 1945 völlig abgesondert war, gab es dennoch einen deutlichen Bedarf. Auch dank der Zagreber Bürger*innenschaft, in der zu dieser Zeit noch immer sehr gut Deutsch gesprochen wurde, gab es eine hohe Anzahl von Besucher*innen, sodass die Lesehalle schnell zu einer großen Leihbibliothek mit einem Bestand von ungefähr 7.000 Büchern anwuchs. So versorgte sie das Publikum vor allem mit österreichischer Literatur, 300 Musikeditionen und 400 Schallplatten. Die Lesehalle organisierte in ihren Räumen jeweils an Dienstag Abenden Vorlesungen, Buch- und Filmabende, Ausstellungen, Konzerte und andere Veranstaltungen, die unter dem Namen „Dienstagabend“ firmierten und ab dem 13. Dezember 1955 stattfanden.

Anhand des jährlichen Berichts ${ }^{25}$ für das Jahr 1957/58 lässt sich nachvollziehen, dass die Lesehalle bereits mehr als 1.500 Mitglieder hatte, von denen täglich 60 die Bibliothek besuchten. Im Jahr 1961 hatte sie bereits 2.347 Mitglieder und im Jahr 1962 wuchs die Zahl dann auf mehr als 2.500 Mitglieder an. Das Jahr 1961 gilt als besonders wichtig für die Lesehalle, weil sie in diesem das Statut bekam, „das ihr ermöglicht, Tätigkeiten auszuführen, die analog zu denen des British Councils oder Institut Français in Jugoslawien waren". ${ }^{26}$ Obwohl sie seit ihrer Gründung die Rolle eines Kulturinstituts hat, wird sie erst im Jahr 1976 in Österreichisches Kulturinstitut umbenannt.

Die hohen Ansprüche, die man im Zusammenhang mit der Vorstellung der österreichischen Kultur an sich selbst gestellt hat, bezeugen auch die Briefe, zu deren Absendern die damals bekanntesten zeitgenössischen österreichischen Schriftsteller zählen: Heimito von Doderer, Elias Canetti (Literatur Nobelpreisträger 1981), Peter Turrini, Peter Handke und viele andere. Außer den Veranstaltungen in ihren eigenen Räumen organisierte die Lesehalle auch Theaterstücke, Konzerte, Ausstellungen 
und Symposien in anderen Institutionen in Zagreb. Im Jahr 2001 wurde das Österreichische Kulturinstitut schließlich in Österreichisches Kulturforum umbenannt, welches vier Jahre später (2005) 3000 eingetragene Benutzer*innen zählte. ${ }^{27}$ Trotz der Tatsache, dass die Bibliothek 1999 überflutet wurde, wobei 500 Bücher zerstört wurden, hatte sie im Jahr 2015 bereits einen Bestand von 15.600 Büchern und 4.600 eingetragene Benutzer*innen. Mit der Zeit sank die Anzahl der aktiven Benutzer*innen, da es nicht mehr länger im Trend war, die kleine Lesehalle im Zentrum der Stadt zu besuchen. Das ging so weit, dass es sich in den Jahren 20102015 gar nur mehr um eine Zahl von nur hundert aktiven Benutzer*innen ${ }^{28}$ handelte, weshalb entschieden wurde, dass die Bibliothek ihr Profil anpassen und sich einem breiteren Zielpublikum zuwenden sollte.

Die „Neugründung“ der Bibliothek erwies sich als eine sehr komplizierte Aufgabe, weil viele Institutionen (wie das Bundesministerium für Europa, Integration und Äußeres der Republik Österreich, die Österreichische Botschaft, die Philosophische Fakultät, die Bibliothek der Philosophischen Fakultät, die Abteilung für Germanistik sowie die Abteilungen für Geschichte, Kunstgeschichte und Komparatistik) beteiligt waren und die damit verbundenen rechtlichen Rahmenbedingungen, technischen Gegebenheiten und unterschiedlichen Interessen in Übereinstimmung gebracht werden mussten. ${ }^{29}$

Zum sechzigsten Jubiläum ihrer Gründung hörte die Bibliothek des Österreichischen Kulturforums somit im Jahr 2015 auf zu bestehen und wurde als Österreich-Bibliothek Zagreb neu gegründet.

Die Österreich-Bibliothek Zagreb wurde am 11. Juni 2015 eröffnet. Den Bestand machten 4.500 Bände aus, die aus der Bibliothek des Österreichischen Kulturforums übernommen wurden und durch eine Spende der GRAWE Versicherung Kroatien für die Beschaffung wertvoller kritischer Ausgaben um 470 Bibliotheksstücke bereichert wurde. Die Sammlung besteht - außer aus Referenzliteratur (eine große Anzahl von Enzyklopädien, Lexika, Wörterbücher und Grammatiken) - aus Primärliteratur (18., 19. und 20. Jahrhundert, Nachkriegsliteratur und Anthologien), Literaturübersetzungen und Sekundärliteratur. Studien und Veröffentlichungen aus den Bereichen der Kulturwissenschaft, Literaturwissenschaft, Sprachwissenschaft, Kultur und Zivilisation, Psychologie, Philosophie, Geschichte, historische Biographien, Kunstgeschichte, Musik, Theater, Film befinden sich ebenso im Bestand wie 130 DVDs mit neueren und alten Filmen aus der Edition Der Standard und 13 Periodika und seit Oktober 2013 darüber hinaus Wochenendausgaben der drei österreichischen Zeitungen: Die Presse, Der Standard und Wiener Zeitung. Die Bibliothek bewirbt Veran- 
staltungen auf ihrer Internetseite (http://oesterreich-bibliothek-zagreb. jimdo.com/) und ihrer Facebook-Seite (https://www.facebook.com/ pages/Austrijska-knji\%C5\%BEnica-Zagreb-\%C3\%96sterreich-Bibliothek-Zagreb/375962885927501).

Die Integration der Altbestände des Kulturforums in die Bibliothek der Philosophischen Fakultät war komplex, weil es sich um eine Bibliothek handelt, deren primäre Rolle es ist, die wissenschaftliche Arbeit an der Fakultät zu unterstützen und Prüfungs- und Seminarliteratur zur Verfügung zu stellen. Bevor das Abkommen zwischen dem Bundesministerium für Europa, Integration und Äußeres der Republik Österreich und der Universität in Zagreb zur Gründung der Österreich-Bibliothek Zagreb unterschrieben wurde, führte man deshalb eine Selektion des Bestandes des Österreichischen Kulturforums durch. Von den 15.000 Bänden wurden jene Bücher, die die wissenschaftliche und Unterrichtstätigkeit fördern, in den Bestand der neugegründeten Bibliothek übernommen, während veraltete oder nur für ein kleineres Publikum relevante Bibliotheksstücke ausgesondert wurden. Bei diesem Vorhaben spielte die Abteilung für Germanistik die Hauptrolle, da sie mit der Bibliotheksverwaltung direkt verhandelte und die Auswahl der Bibliotheksstücke sowie die Gestaltung des Programms der Österreich-Bibliothek maßgeblich beeinflusste.

Der Aufbau der Sammlungen innerhalb der Bibliothek der Philosophischen Fakultät folgt gewissen Regeln, wobei auch die Österreich-Bibliothek Zagreb keine Ausnahme darstellt. Für ihren Bestand stellte die Philosophische Fakultät einen Teil der Regale im Freihandbereich, im Bibliotheksmagazin, in anderen Räumlichkeiten der Fakultät und im Bibliotheksfoyer für ihre kulturellen und wissenschaftlichen Tätigkeiten zur Verfügung. Sie sorgt für die Integrität der Sammlung, ihre Aufstellung, fachliche Bearbeitung, Zuweisung einer wissenschaftlichen und bibliothekarischen Leitung und hat die Österreich-Bibliothek Zagreb den anderen Sammlungen der Fakultätsbibliothek so angeglichen (mehr als 30 Sammlungen mit über 600.000 Bibliotheksstücken, über 9.000 registrierte Benutzer*innen und mehr als 200.000 Transaktionen hinsichtlich der Ausleihe, Vorbestellung und Verlängerung der Leihfrist jährlich), ${ }^{30}$ dass alle Bürger*innen die Medien benutzen sowie die Studierenden und Fakultätsangehörigen diese auch ausleihen können.

Die Österreich-Bibliothek Zagreb ist der Bibliothek der Philosophischen Fakultät seitens des BMEIA als eine unteilbare Sammlung ausgeliehen, die sich im Besitz der Republik Österreich befindet. Das BMEIA sorgt gemäß seiner budgetären Möglichkeiten für den Erwerb neuer Medien und ermöglicht den Mitarbeiter* innen einen Aufenthalt in der Dauer von vier Wochen 
pro Jahr in Österreich für fachliche und wissenschaftliche Zwecke. Bei der Gestaltung der Sammlung und der Organisation von Veranstaltungen bekommt die Bibliothek Hilfe von einem österreichischen Lektor und dem Österreichischen Kulturforum Zagreb. ${ }^{31}$

In Zusammenarbeit mit dem Österreichischen Kulturforum werden Konferenzen, Gastvorlesungen, Workshops, Buchvorstellungen, Wettbewerbe für Studierende, Filmabende, Ausstellungen und ähnliches veranstaltet.

\subsection{Ausleihen der Österreich-Bibliothek Zagreb}

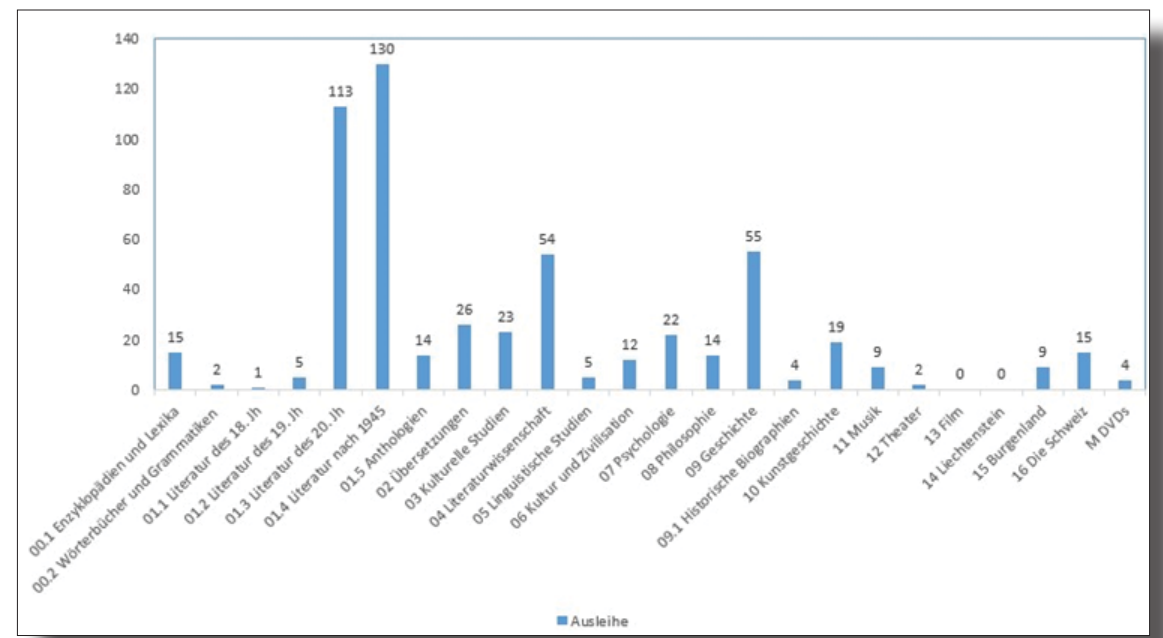

Abb. 4: Ausleihen der Österreich-Bibliothek Zagreb nach Fachgruppen

Dank des integrierten Bibliothekssystems $\mathrm{KOHA}$, das in der Bibliothek der Philosophischen Fakultät eingesetzt wird und „die komplette Bibliotheksverwaltung umfasst, darunter auch einen öffentlich zugänglichen Katalog, der verschiedene Web 2.0-Features - wie zum Beispiel Tagging, eine Kommentarfunktion, Soziale Netzwerke und RSS-Quellen - anbietet, die Erstellung einer persönlichen Seite in dem öffentlichen Katalog und einer eigenen Literaturliste, die Anpassung der Suche im Katalog; Ausleihe und Administration der Benutzer*innen; ein Modul für die Erwerbung; Unterstützung für mehrere Bibliotheken, die das gleiche System benutzen, Betreuung der Periodika sowie ein Modul für Berichte und andere Werkzeuge, die die Verwaltung der Bibliothek unterstützen" 32 , konnte die Systembibliothekarin spezifische Daten über die Ausleihe im Zeitraum vom 11. Juni 2015 bis zum 
20. April 2017 erheben (Abbildung 4) ${ }^{33}$. In diesem Zeitraum wurden insgesamt 603 Bibliotheksstücke ausgeliehen - 549 Bücher und 4 DVDs aus dem Freihandbereich sowie 50 Bücher aus dem Bibliotheksmagazin. Aus Platzgründen befindet sich ein Teil des Bestandes im Bibliotheksmagazin (u.a. Dubletten und Rara). Die ausgewerteten Daten haben bestätigt, was auch in der Praxis zu bemerken war. Die Österreich-Bibliothek Zagreb dient als Ergänzung zur Germanistiksammlung, die „1904 als Handbibliothek des Seminars für Deutsche Philologie gegründet wurde. " ${ }^{34}$ Die Österreich-Bibliothek Zagreb beinhaltet Bibliotheksstücke, die entweder in dem Bestand der germanistischen Sammlung nicht vorhanden sind oder an denen es in diesem mangelt; so zum Beispiel österreichische Primärliteratur aus der ersten und zweiten Hälfte des 20. Jahrhunderts sowie insbesondere moderne österreichische Literatur, was man auch aus den Daten über die Ausleihe von 113 literarischen Werken aus der ersten Hälfte des 20. Jahrhunderts, 130 literarischen Werken aus der zweiten Hälfte des 20. Jahrhunderts, 54 Werken über Literaturwissenschaft (Literaturgeschichte, Literaturtheorie, Biographien, Interpretationen österreichischer Schriftsteller), 55 Werken zur Geschichte von Österreich und 50 Werken aus dem Bibliotheksmagazin, wobei es sich um literarische Primärwerke aus der ersten Hälfte des 20. Jahrhunderts handelt, bemerken kann.

\subsection{Die Beziehung zwischen der Österreich-Bibliothek Zagreb und ihrer Trä- gerinstitution}

Jede Sammlung, die heute den Studierenden und Professor*innen in der Bibliothek der Philosophischen Fakultät zur Verfügung steht, ist Resultat eines langjährigen Bestehens, Wirkens und Arbeitens vieler Seminarbibliotheken.

Gründung und Aufbau neuer Sammlungen erfolgt in jeder Fakultätsbibliothek schrittweise. Der Bestand wird nach und nach aufgebaut, gemäß den Anforderungen, die das Studienprogramm stellt, oder auch oft wegen der Etablierung eines neuen Studienprogramms und als Resultat einer Abmachung zwischen der Bibliothek und den Professor*innen, welche die neuen Fächer betreuen. Jede neue Sammlung muss thematisch und inhaltlich das Gebiet einer Studiengruppe oder eines Studienprogrammes abdecken, die an der Philosophischen Fakultät in Zagreb aufgeführt sind und studiert werden können. Durch die langjährige Geschichte des bibliothekarischen Wirkens an der Philosophischen Fakultät folgt ihre Bibliothek auch der Tradition einer Hochschulbibliothek mit dem Schwerpunkt auf dem Gebiet der Geistes-, Sozial- und Kulturwissenschaften, aber auch darüber hinaus. 
Die Österreich-Bibliothek Zagreb hat auf eine ganz divergente Art mit der Arbeit innerhalb der Bibliothek der Philosophischen Fakultät angefangen. Es handelt sich um eine unteilbare Sammlung, die sich im Besitz der Republik Österreich befindet und auf unbeschränkte Zeit an die Bibliothek der Philosophischen Fakultät verliehen wurde. Wie auch die anderen 65 Österreich-Bibliotheken rund um die Welt wird sie vom Bundesministerium für Europa, Integration und Äußeres der Republik Österreich (BMEIA) finanziert, welches das auch als ihr Träger wirkt. Die Bibliothek der Philosophischen Fakultät wurde zum Standort der Österreich-Bibliothek Zagreb, die ihr einen Standort, fachliche Bearbeitung, einen wissenschaftlichen Betreuer und eine Bibliothekarin zur Verfügung stellt, wobei die Ausleiheregeln die gleichen sind wie für alle anderen Sammlungen der gesamten Bibliothek.

Der Prozess der Aufnahme einer neuen Sammlung in den Rahmen ihrer Fakultätsbibliothek wurde zwischen der Leitung der Philosophischen Fakultät und des Österreichischen Kulturforums vereinbart. In den Standards für Hochschulbibliotheken in Kroatien steht, dass „Die Fakultätsbibliothek und die ihr angehörigen Abteilungsbibliotheken, beziehungsweise der zentrale Bibliotheksdienst der Fakultät und die ihr angehörigen Abteilungsbibliotheken, sind als fachliche Organisationseinheiten aufgebaut, deren Pläne, Programme und Berichte der Rat der Wissenschaftler und Lehrenden bewilligt und bewertet und danach der zuständigen Behörde und der zentralen Universitätsbibliothek weiterleitet“. Der Bibliotheksrat hatte im Gründungsprozess der Österreich-Bibliothek Zagreb keinen Freiraum irgendwelche Fragen zu stellen. Der für die Hochschulbibliotheken zuständige Dienst an der National- und Universitätsbibliothek Zagreb, der für den Betrieb der Bibliothek zuständig ist, wurde nicht darüber benachrichtigt, dass der Bibliothek der Philosophischen Fakultät eine neue Sammlung zugeteilt werden sollte. ${ }^{35}$

Die Gründung der Österreich-Bibliothek Zagreb brachte der Fachbibliothekarin, die für zwei weitere Sammlungen in der Bibliothek der Philosophischen Fakultät zuständig ist, einen weiteren Kompetenzbereich ein. Die Germanistiksammlung ist die zweitgrößte Sammlung in der Bibliothek der Philosophischen Fakultät, daneben betreut sie auch die aus der Germanistiksammlung ausgesonderte Nederlandistiksammlung (Wahlfach bzw. später Studienprogramm mit eigenem Lehrstuhl). Die Fachbibliothekarin erledigt mithilfe studentischer Hilfskräfte, die unter Aufsicht eines Bibliothekars bei der fachlichen Bearbeitung und Bewahrung der Bibliotheksstücke teilnehmen, alle dazugehörigen Vorgänge (Erwerb, Inventarisierung, formale und fachliche Bearbeitung). ${ }^{36}$ Die Österreich-Bibliothek Zagreb 
fordert darüber hinaus auch die Organisation zahlreicher Veranstaltungen, die Kuratierung von Internet- und Facebook-Seiten, aber bietet im Gegenzug auch zusätzliche Möglichkeiten zur Zusammen- mit bzw. Mitarbeit an österreichischen kulturellen und wissenschaftlichen Institutionen. Die Bibliothek der Philosophischen Fakultät hat damit eine ganz neue Dimension, Aufgabe und Pflicht übernommen. Außer der Verpflichtung gegenüber den Bedürfnissen ihrer primären akademischen Benutzer*innengruppe, Wissenschaftler*innen auf dem Gebiet der Geistes-, Sozial- und Kulturwissenschaften, sowie hinsichtlich der Inhalte, die innerhalb der Studiengruppen der Philosophischen Fakultät gelehrt und beforscht werden, muss sie auch die Angelegenheiten der Österreich-Bibliothek Zagreb abdecken und verbreitern.

\section{Schlussbemerkungen}

Bis heute wurden 65 Österreich-Bibliotheken in 28 Ländern rund um die Welt eröffnet. Die erste solche Gründung erfolgte 1986 in Krakau. In weiterer Folge hat das Außenministerium der Republik Österreich seit 1989 ein Programm für Deutschlektor*innen an Universitäten auf dem Gebiet der ehemaligen Habsburgermonarchie lanciert, mit dem Ziel die deutsche Sprache zu verbreiten und die kulturellen Beziehungen mit Österreich zu stärken. Da Österreichische Kulturforen ausschließlich in Hauptstädten gegründet werden, werden in Städten und Universitätszentren ohne Österreichische Kulturforen zur Unterstützung ihres Programms zusätzlich Bibliotheken eröffnet. Deshalb ist es auch nicht verwunderlich, dass sich der größte Anteil der Österreich-Bibliotheken in der Tschechischen Republik, Polen, Ungarn und Kroatien befindet, und zwar an Universitäts- und Fakultätsbibliotheken und seltener an öffentlichen Bibliotheken angesiedelt. Alle Österreich-Bibliotheken gelten als unteilbare Sammlungen, die sich im Besitz des Bundesministeriums für Europa, Integration und Äußeres der Republik Österreich (BMEIA) befinden, das ihr Unterhaltsträger ist und den Aufbau der Sammlungen finanziert, während sich die Gastinstitutionen der Bibliotheken um Infrastruktur und Personal kümmern. Die Bibliotheksbestände haben den Status von Dauerleihgaben. Die Sektion $\checkmark$ (Kultur) des österreichischen Bundesministeriums für Europa, Integration und Äußeres leitet mit Unterstützung der lokalen Botschaften und österreichischen Kulturforen die Organisation und Koordination der Österreich-Bibliotheken und nimmt auf diese Weise an der Organisation der Veranstaltungen innerhalb der Bibliothek teil, bringt alle Österreich-Biblio- 
theken und ihre Mitarbeiter mithilfe einer digitalen Vernetzungsplattform Österreich-Bibliotheken im Ausland (http://www.oesterreich-bibliotheken. at/) zusammen, was auch im Bericht ${ }^{37}$ für das Jahr 2015 deutlich wird. Der gesamte Bestand der Österreich-Bibliotheken zählt derzeit 432.000 Bibliotheksstücke. Die Österreich-Bibliotheken haben ungefähr 380.000 Besucher*innen pro Jahr und organisieren ca. tausend verschiedene kulturelle und wissenschaftliche Veranstaltungen. Die zahlreichen Veranstaltungen der Österreich-Bibliothek ziehen nicht nur Germanistikstudent*innen und ihre Professor*innen an, sondern auch Studierende anderer Studienrichtungen sowie Menschen, die nicht direkt mit der Philosophischen Fakultät verbunden sind. Auf diese Weise werden Kontakte und Freundschaften geschlossen, die in gemeinsamen neuen Projekten resultieren können.

\section{MMag. ${ }^{a}$ Blaženka Klemar Bubić Österreich-Bibliothek Zagreb E-Mail: bklemar@ffzg.hr}

\section{Literatur}

Glovacki-Bernardi, Zrinjka; Hölbing Matković, Lara; Petrušić-Goldstein, Sanja (Hgg): Agramer rječnik njemačkih posuđenica u zagrebačkom govoru. Zagreb: Novi Liber, 2013.

Die Bibliotheken: Landkarte. Österreich-Bibliotheken im Ausland. https:// www.oesterreich-bibliotheken.at/oesterreich-bibliotheken/die-bibliotheken/landkarte/ (Zugriff: 16.3.2019)

Die Bibliotheken: Bibliotheksverzeichnis. Österreich-Bibliotheken im Ausland. https://www.oesterreich-bibliotheken.at/oesterreich-bibliotheken/diebibliotheken/oesterreichische-bibliotheken-bibliotheken-mit-oesterreich-bezug/ (Zugriff: 16.3.2019).

Erl Šafar, Marija. Knjižnica u multikulturalnoj sredini-Austrijska čitaonica Gradske i sveučilišne knjižnice Osijek. In: 4. međunarodni stručni skup Knjižnica središte znanja i zabave : zbornik radova. Hg. Frida Bišćan. Karlovac: Gradska knjižnica „Ivan Goran Kovačić“ 2008, S. 95-101.

Glavica, Marijana: Austrijska knjižnica - posudba. Privatnachricht (21.4.2017).

Karlović, Zvjezdana: Korisnici Austrijske knjižnice. Privatnachricht (19.4.2017).

Koha Croatian user group. https://saturn.ffzg.hr/koha-cug/index.cgi (Zugriff: 21.4.2017). 
Kulaš, Vanja. Institut demonstratora u Knjižnici Filozofskog fakulteta u Zagrebu. »HKD-Novosti« 63(2014). http://www.hkdrustvo.hr/hkdnovosti/clanak/912 (Zugriff: 21.4.2017).

Lacko Vidulić, Svjetlan: Tekst o Austrijskoj knjižnici. Privatnachricht (24. 4. 2017).

Obad, Vlado: Roda Roda und die deutschsprachige Literatur aus Slawonien. Wien; Köln; Weimar: Böhlau 1996.

Stamać, Truda (Hg.): Od utorka do utorka : Austrijski kulturni institut/forum u vlastitim dokumentima : spomen spis u povodu jubileja Austrijskog kulturnog foruma. Zagreb: Durieux 2006.

Pavić Pintarić, Anita. Austrijska knjižnica Zadar. Privatna poruka. (13.4.2017.)

Popis stanovništva kućanstva i stanova 2011. Stanovništvo prema narodnosti po gradovima/općinama. http://www.dzs.hr/Hrv/censuses/census2011/ results/htm/H01_01_04/h01_01_04_RH.html (Zugriff: 21.4.2017).

Standardi za visokoškolske knjižnice u Republici Hrvatskoj. »Vjesnik bibliotekara Hrvatske« 33, 1-4(1990) S. 201-209.

Stojić, Aneta. Austrijska knjižnica Rijeka. Privatna poruka. (6.4.2017).

Pravilnik o matičnoj djelatnosti knjižnica u Republici Hrvatskoj. »Narodne novine« 43/2001. http://narodne-novine.nn.hr/clanci/sluzbeni/2001_05_43_715.html (Zugriff: 1.11.2017).

Sporazum [između Saveznog ministarstva za Europu, integraciju i međunarodne poslove Republike Austrije i Sveučilišta u Zagrebu o osnivanju Austrijske knjižnice Zagreb]. (11. lipnja 2015.) Klasa: 74006/15-01/34 Ur.br.: 3804-15-1.

Stanarević, Snježana; Petković, Siniša; Lacović, Darko. Petnaest godina djelovanja Austrijske čitaonice pri Gradskoj i sveučilišnoj knjižnici Osijek. In: 6. međunarodni stručni skup Knjižnica - središte znanja i zabave : zbornik radova. Hg. Frida Bišćan. Karlovac: Gradska knjižnica „Ivan Goran Kovačić“ 2008, S. 30-41.

Todorčev Hlača, Katarina: Strategija razvoja središnjih knjižnica nacionalnih manjina unutar mreže narodnih knjižnica. »Vjesnik bibliotekara Hrvatske« 58, 1-2(2015), S. 45-66.

Über uns: Geschichte. Österreich-Bibliotheken im Ausland. http://www. oesterreich-bibliotheken.at/ (Zugriff: 19.1.2017).

Sammlung Germanistik. http://www.ffzg.unizg.hr/german/cms/index. php?option=com_content\&task=view\&id=12\&ltemid=29 (Zugriff: 12.4.2017). 
1 Klemar Bubić, B., Luić-Vudrag, D. (2017). Austrijska knjižnica na Filozofskom fakultetu u Zagrebu. Vjesnik bibliotekara Hrvatske, 60(1), 205220. ins Deutsche übersetzt von Lorena Martinić.

2 Vgl. Glovacki-Bernardi/Höbling Matković/Perušić-Goldstein (Hgg.): Agramer, rječnik njemačkih posuđenica u zagrebačkom govoru, S. 6.

3 Ebd.

4 http://www.dzs.hr/Hrv/censuses/census2011/results/htm/H01_01_04/ h01_01_04_RH.html

5 Todorčev Hlača: Strategija razvoja središnjih knjižnica nacionalnih manjina unutar mreže narodnih knjižnica, S. 49.

6 Karlović: Privatnachricht am 19.4.2017.

7 Standardi za visokoškolske knjižnice u Republici Hrvatskoj, S. 201.

8 Ebd.

9 Ebd.

10 http://www.oesterreich-bibliotheken.at

11 Ebd.

12 https://www.oesterreich-bibliotheken.at/oesterreich-bibliotheken/diebibliotheken/landkarte/

13 https://www.oesterreich-bibliotheken.at/oesterreich-bibliotheken/diebibliotheken/oesterreichische-bibliotheken-bibliotheken-mit-oesterreich-bezug/

14 Ebd.

$15 \mathrm{Vgl}$. Stanarević/Petković/Lacović. Petnaest godina djelovanja Austrijske čitaonice pri Gradskoj i sveučilišnoj knjižnici Osijek, S. 35.

16 Ebd., S. 34.

17 Erl Šafar: Knjižnica u multikulturalnoj sredini-Austrijska čitaonica Gradske i sveučilišne knjižnice Osijek, S. 98.

18 Todorčev Hlača: Strategija razvoja središnjih knjižnica nacionalnih manjina unutar mreže narodnih knjižnica, S. 49.

19 Vgl. Obad: Roda Roda und die deutschsprachige Literatur aus Slawonien, S. 9.

20 Vgl. Stanarević/Petković/Lacović. Petnaest godina djelovanja Austrijske čitaonice pri Gradskoj i sveučilišnoj knjižnici Osijek, S. 35.

21 Ebd., str. 37.

22 Ebd.

23 Stojić : Privatnachricht am 6.4.2017.

24 Pavić Pintarić: Privatnachricht am 13.4.2017.

25 Stamać Truda (Hg.): Od utorka do utorka : Austrijski kulturni institut/forum u vlastitim dokumentima : spomen spis u povodu jubileja Austrijskog kulturnog foruma, S. 12. 
26 Ebd., S. 17.

27 An dieser Stelle möchten wir uns bei der Bibliothekarin des Österreichischen Kulturforums Zvjezdana Karlović bedanken, die uns diese Daten zur Verfügung gestellt hat.

28 Ebd.

29 Vgl. Lacko Vidulić: Privatnachricht am 24. 4. 2017.

30 Ebd.

31 Vgl. Sporazum [između Saveznog ministarstva za Europu, integraciju i međunarodne poslove Republike Austrije i Sveučilišta u Zagrebu o osnivanju Austrijske knjižnice Zagreb]. (11. juni 2015) Klasa: 740-06/1501/34 Ur.br.: 3804-15-1.

32 https://saturn.ffzg.hr/koha-cug/index.cgi

33 Glavica: Privatnachricht am 21.4.2017.

34 http://www.ffzg.unizg.hr/german/cms/index.php?option=com_conte nt\&task=view\&id=12\&ltemid $=29$

35 Sukladno odredbama Zakona o knjižnicama (»Narodne novine« broj 105/97, 5/98 i 104/00), čl.31 stavak 3. donesen je Pravilnik o matičnoj djelatnosti knjižnica u Republici Hrvatskoj prema kojem NSK Zagreb sudjeluje u unapređivanju, organiziranju, osiguravanju i podršci kvalitetnom radu visokoškolske knjižnice. [citirano: 2017-11-1]. Dostupno na. http://narodne-novine.nn.hr/clanci/sluzbeni/2001_05_43_715.html

36 Kulaš: Institut demonstratora u Knjižnici Filozofskog fakulteta u Zagrebu.

37 http://www.oesterreich-bibliotheken.at/verzeichnis.php 